\title{
Biocontrol of mycotoxins: dynamics and mechanisms of action
}

\begin{abstract}
This paper discusses the relationship between biocontrol agents (BCAs) and mycotoxigenic fungi and mycotoxin control. In most cases BCAs are examined for control of growth of fungal pathogens and disease symptoms. However, for mycotoxin control the approach and focus needs to be different. The mechanism of action and the inoculum dose necessary for control of toxin production by Aspegillus, Penicillium and Fusarium species may be different from that for traditional fungal plant pathogens. The mechanisms of action, the relative inoculum potential and the impact that interacting environmental conditions have on control of key components of the life cycle of mycotoxigenic fungi are considered. The practical aspects of production and formulation hurdles are discussed and potential future approaches and strategies which may need to be considered for more effective biocontrol of mycotoxigenic fungi and mycotoxins are presented.
\end{abstract}

Keyword: Biocontrol agents (BCAs); Mycotoxigenic fungi; Mycotoxin control 